\title{
HVS Inspired System for Script Identification in Indian Multi-script Documents
}

\author{
Peeta Basa Pati ${ }^{\star}$ and A.G. Ramakrishnan \\ Department of Electrical Engineering, \\ Indian Institute of Science, Bangalore - 560 012, India \\ pati@ee.iisc.ernet.in
}

\begin{abstract}
Identification of the script of the text, present in multi-script documents, is one of the important first steps in the design of an OCR system. Much work has been reported relating to Roman, Arabic, Chinese, Korean and Japanese scripts. Though some work has already been reported involving Indian scripts, the work is still in its nascent stage. For example, most of the work assumes that the script changes only at the level of the line, which is rarely an acceptable assumption in the Indian scenario. In this work, we report a script identification algorithm, which takes into account the fact that the script changes at the word level in most Indian bilingual or multilingual documents. Initially, we deal with the identification of the script of words, using Gabor filters, in a biscript scenario. Later, we extend this to tri-script and then, five-script scenarios. The combination of Gabor features with nearest neighbor classifier shows promising results. Words of different font styles and sizes are used. We have shown that our identification scheme, inspired from the Human Visual System (HVS), utilizing the same feature and classifier combination, works consistently well for any of the combination of scripts experimented.
\end{abstract}

Keywords: Gabor filter, script identification, prototype selection.

\section{Introduction}

India has 26 official languages represented with 13 unique scripts. Many official documents are multi-script in nature. Besides, there are other Asian countries, where multi-script documents exist. Thus, identification of the script is one of the necessary challenges for the designer of OCR systems dealing with such multi-script documents. Quite a few results have been reported in the literature, identifying the scripts in multi-script documents. However, very few of these works deal with script identification at the word level.

Spitz et al. [1] use the spatial relationship between the structural features of characters for distinguishing Han from the Latin script. Asian scripts (Japanese, Korean and Chinese) are differentiated from Roman by an uniform vertical distribution of upward concavities. In the case of the above Asian scripts, the measure

\footnotetext{
* Corresponding author.
} 
of optical density (i.e. the number of ON-pixels per unit area) is employed to distinguish one from the other. Hochberg et al. 22 use cluster based templates for script identification. They consider thirteen different scripts including Devanagari, an Indian script used to write Hindi, Sanskrit, Marathi and Nepali languages. They cluster the textual symbols (connected components) and create a representative symbol or a template for each cluster. Identification is through comparison of textual symbols of the test documents with those of the templates. However, the requirement of the extraction of connected components makes this feature a local one. Wood et al. [3] suggest a method based on Hough transform, morphological filtering, and analysis of projection profile. Their work involves the global characteristics of the text.

Tan [4] has suggested a method for identifying six different scripts using a texture based approach. Textual blocks of $128 \times 128$ are taken and filtered with angular spacings of $11.25^{\circ}$. This method assumes that the script of the text changes at the paragraph level. Roman, Persian, Chinese, Malayalam, Greek and Russian scripts, with multiple font sizes and styles (font invariance within the block), are identified.

Pal and Chaudhuri [5] have proposed a method, based on a decision tree, for recognizing the script of a line of text. They consider Roman, Bengali and Devanagari scripts. They have used the projection profile, besides statistical, topological and stroke-based features. At the initial level, the Roman script is isolated from the other two, by examining the presence of the headline 1 (shirorekha). Devanagari is differentiated from Bangla by identifying the principal strokes [5. In [6], they have extended their work to the identification of the script from a given triplet. Here, they have dealt with almost all the Indian scripts. Besides the headline, they have used some script-dependent structural properties, such as the distribution of ascenders and descenders, the position of the vertical line in a text block, and the number of horizontal runs.

Chaudhuri and Seth [7] have proposed a technique using features such as the horizontal projection profile, Gabor transform and aspect ratio of connected components. They have handled Roman, Devanagari, Telugu and Malayalam scripts.

For most of the above reported works, the textual block taken for the script recognition task is either a mono-script line or a paragraph. However, in the Indian context, in the official documents, technical reports, magazines and application forms, the script could, in principle, vary at the word level. Figure 1 demonstrates this with an example bilingual document containing Devanagari and Roman scripts.

Recognition of the script, using statistical features, at word level has been reported by Dhanya et al. [8]. Here the authors tried to differentiate the Tamil and Roman scripts using various feature-classifier combinations. This work has been extended by Pati et al. 9]. Here the authors have identified Odiya and Devanagari scripts, besides Tamil, against the Roman script. They use a bank of Gabor

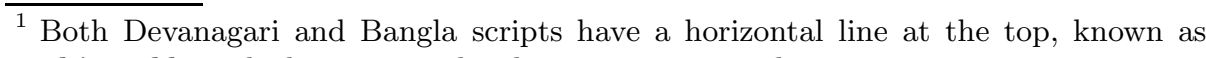
shirorekha, which connects the characters in a word. 


\section{पढ़ें और फिर किताबें पढ़ के एक लेक्चर दें। I am not lecturer कई कई लोग कहते, आज जी उनका Lecture होगा। मुझे बड़ा अजीब लगे। ये Lectures क्या हुआ भई? Lectures are prepared by lecturers. \\ यहाँ कोई आये हो कालेज Professor या स्कूल के Teacher स्कूल के टीचर को भी आज कल तैयारी करनी पड़ती है लेकिन कालेज के प्रोफेसर को, University के प्रोफेसर को एक Subject के ऊपर बोलने से पहले तैयारी करनी पड़ती, पढ़ना पड़ता है। Latest news क्या हैं? उनका सबका Viva करना पड़ता है। पर मैरे लिए यहाँ आ के बैठना ओर बोलना बस यही तैयारी कि यहाँ आ के बैठ गये।}

Fig. 1. A sample bi-script document showing interspersed Hindi and English words

filters for feature extraction with linear discriminant classifier for decision making. Besides, redesign of the Gabor functions has allowed to enhance the system efficiency. Pal and Chaudhury [5] have tried to discriminate Roman, Devanagari and Bangla scripts at the word level using a set of structural features and a tree based classifier. They have extended their approach to identify scripts in another triplet, Roman, Devanagari and Telugu scripts 10. Padma and Nagabhushan [11] have discriminated Roman, Devanagari and Kannada script on similar lines.

In the present work, we significantly extend our earlier work [8,9] by refining the parameters of the Gabor filters based on our experiments, and also experimenting on a much larger database of words. One of the motivations is to come out with a technique that works for any pairs of scripts, since in the border areas between different states in India, two languages, and hence, two scripts co-exist. Further, since Hindi is the national language, which uses the Devanagari script, we explore the effectiveness of our approach in dealing with documents that use a state language, English and Hindi. Thus, we have evaluated the performance of our technique for recognizing three scripts, namely a local script, Devanagari and Roman. In addition, we also determine how effectively we can determine the script of a word without any a priori knowledge except that it is one of the 5 possible scripts.

\section{Data Description}

Our database consists of about 1000 scanned documents of different scripts. These documents are scanned from various sources such as (i) laser printed pages, (ii) printed books, (iii) magazines, (iv) newspapers and (v) official documents. We have collected about 4500 words each from Tamil, Kannada, Odiya, Devanagari and Roman scripts [12. 3000 of these are selected randomly for each script, to form the training set, while the rest 1500 words form the test set. Most of these words have been segmented and collected from actual bilingual documents, with the script varying at the word level. We have assumed that a word contains at least two characters. These words contain a lot of variability in 
terms of font size and style, as well as the age and nature of the document from which they are collected. We ensure that there are no blank rows or columns at the boundaries of the word image.

\section{System Description}

Human Visual System (HVS) is best modeled by Gabor function:2 [15, because the mammalian visual cortex involves a set of parallel filter channels, which are quasi-independent [16] as well as direction dependent [17. This supports the theory of multi-channel filtering of signals in systems that model biological vision [18. This scheme has been successful in texture segmentation [19] and researchers have also used it for text page segmentation [20, 21.

There are two approaches taken for script identification in a multi-script scenario. One of them extensively studies the similarities and differences in the structures between the co-occurring scripts while the second method deals with each script as a different texture. In our view, the latter method is more robust as it deals with the script regardless of the size or style of the font. Thus, we use a multi-channel filtering approach, employing Gabor functions, for script identification. This claim of ours is supported by earlier work on page layout analysis [21] and script identification [8, 9].

The Gabor filter bank used has three different radial frequencies $(0.125,0.25$ $\& 0.5)$ and six different angles of orientation $(\theta=0,30,60,90,120$ and 150 degrees). Thus, we have used a radial frequency bandwidth of 1 octave and an angular bandwidth of $30^{\circ}$. The spatial spread of each filter along the $x-$ and $y$-coordinates are determined by the standard deviations of the Gaussians, $\sigma_{x}$ and $\sigma_{y}$, respectively. Both of them are functions of the radial frequency and angular bandwidth. The three radial frequencies with six $\theta$ 's give a combination of 18 odd and 18 even filters. The size of each filter mask is $13 \times 13$. Each of the word images, without any size normalization, is convolved in the spatial domain with these filters. A 36-dimensional feature vector is formed with the total energy in each of the filtered images forming a feature.

We use linear discriminant (LD) and nearest neighbor (NN) classifiers as the decision makers. Each feature vector acts as a point in a $d$-dimensional space, where $d(=36)$ is the dimensionality of the feature vector. The NN classifier finds the closest neighbor of the test pattern in this space. It then assigns the class value of this closest pattern to the test pattern. Thus, in a bi-class case, if the test pattern has a closest neighbor from class $\omega_{1}$, then the test pattern is assumed to be from class $\omega_{1}$. In LDC, however, we try to find a hyper-plane which best discriminates the classes in this $d$-dimensional space. In a bi-class case, this hyper-plane divides the feature space into two parts such that the training patterns of the two different classes lie on the opposite sides of this plane. Thus, when a test pattern lies on one side of the hyper-plane, it gets the class value meant for that side of the hyper-plane. A multi-class scenario

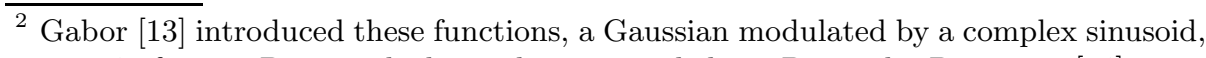
in 1946 for one-D case which was later extended to 2D case by Daugman [14]. 
could be considered to be consisting of a number of 2-class scenarios, for LDC evaluation. Thus, by combining the 36-dimensional Gabor features with the LDC or NN classifier, we try to identify the script of a word in bi-script, tri-script and five-script cases.

3000 training patterns is a fairly large set per script. Moreover, quite a lot of samples could be actually very closely placed in the feature space. For deciding the discriminating hyper-plane, only few representing samples which lie on the border of the class are sufficient. Besides, a large training set consumes a lot of computational time while the NN classifier is at work. Thus, it is worth looking at prototype selection mechanisms, where a reduced set called a prototype set takes the place of the training or reference set. This being a small set has the advantage of combined space and computational time efficiency.

Susheela Devi and M N Murthy 22] have proposed an efficient incremental prototype set building algorithm. They have tested their algorithm over a variety of benchmark data sets and reported that it performs consistently. This algorithm has two stages: (i) set growing stage and (ii) set reduction stage. While the set growing stage increases the number of prototypes till all the training samples are recognized, the set reduction technique removes those prototypes which are responsible only for self-classification. We have taken the training sets of all the five scripts and employed a NN classifier for this purpose. We, however, have used only the set growing technique and terminate the process when it achieves $100 \%$ recognition accuracy of the training set against the prototype set, for all the five classes.

\section{Results}

To confirm that our selection of test and training sets are class representative in nature, we tested the accuracy of recognition of the test set against that of the training and vice versa. We use the NN classifier for this purpose. The test set recognition accuracy against the train set is $96.0 \%$ while that of the train set against the test set is $94.7 \%$. This shows that the selection of the test and train sets are fairly independent and are class representative in nature.

The prototype selection mechanism was able to reduce the training set to a much smaller set. At the end of the process, we had 291, 290, 258, 570, 485 samples representing the Roman, Devanagari, Kannada, Odiya and Tamil scripts, respectively. Thus, we had a total of only 1894 samples representing 15000 training samples of all the five classes, saving about $87 \%$ of memory and computation.

The five scripts we considered for our experiments result in 10 different biclass problems. We report the accuracies of the bi-class script identification in Table 1. The performance is presented in percentage and give the average recognition accuracies of both the scripts involved. We are presenting the accuracies of the LD and NN classifiers together. In the table, the characters R, D, K, O and $\mathrm{T}$ represent the Roman, Devanagari, Kannada, Odiya and Tamil scripts, respectively. It can be observed that both the LD and NN classifiers have performed very well with the Gabor feature vectors, though in most of the cases 
Table 1. Recognition rate for pairs of scripts involving various Indian scripts. The figures indicate the average correct recognition of words in both scripts of the test set against the train set and the prototype set using NN or LD classifiers. (R:Roman, D:Devanagari, K:Kannada, O:Odiya and T:Tamil).

\begin{tabular}{|c|c|c|c|c|c|c|c|c|c|c|c|c|}
\hline & \multicolumn{5}{|c|}{ TRAIN SET } & \multicolumn{5}{|c|}{ PROTOTYPE SET } \\
\hline & & & $\mathrm{R}$ & $\mathrm{D}$ & $\mathrm{K}$ & $\mathrm{O}$ & $\overline{\mathrm{T}}$ & $\mathrm{R}$ & $\mathrm{D}$ & $\mathrm{K}$ & $\mathrm{O}$ & $\mathrm{T}$ \\
\hline \multirow{10}{*}{$\begin{array}{r}\text { TRAIN } \\
\text { SET }\end{array}$} & \multirow{5}{*}{ LDC } & $\mathrm{R}$ & - & 99.1 & 99.2 & 97.8 & 97.4 & - & 98.7 & 98.7 & 97.6 & 96.0 \\
\hline & & $\bar{D}$ & 99.1 & - & 99.5 & 99.1 & 98.9 & 98.7 & \begin{tabular}{|l|}
- \\
\end{tabular} & 99.0 & 99.1 & 98.4 \\
\hline & & $\bar{K}$ & $\overline{99.2}$ & 99.5 & - & 98.0 & 99.2 & 98.7 & 99.0 & - & 97.1 & $\overline{99.2}$ \\
\hline & & $\mathrm{O}$ & $\overline{97.8}$ & 99.1 & 98.0 & -1 & 98.2 & 97.6 & 99.1 & 97.1 & - & 97.8 \\
\hline & & $\mathrm{T}$ & $\overline{97.4}$ & 98.9 & 99.2 & 98.2 & - & 96.0 & 98.4 & 99.2 & 97.8 & - \\
\hline & \multirow{5}{*}{$\mathrm{NNC}$} & $\overline{\mathrm{R}}$ & $\overline{-}$ & 99.4 & $\overline{99.6}$ & 98.5 & 98.8 & - & 98.9 & 99.5 & 97.4 & 97.6 \\
\hline & & $\bar{D}$ & $\overline{99.4}$ & - & 99.7 & 99.4 & 99.2 & 98.9 & - & 99.5 & 98.8 & 98.0 \\
\hline & & $\mathrm{K}$ & 99.6 & 99.7 & - & 98.2 & \begin{tabular}{|l|}
99.4 \\
\end{tabular} & 99.5 & 99.5 & - & 96.2 & 99.0 \\
\hline & & $\bar{O}$ & 98.5 & 99.4 & 98.2 & \begin{tabular}{|l|}
- \\
\end{tabular} & 97.3 & 97.4 & 98.8 & 96.2 & - & 95.6 \\
\hline & & $\overline{\mathrm{T}}$ & 98.8 & 99.2 & 99.4 & 97.3 & - & 97.6 & 98.0 & 99.0 & 95.6 & - \\
\hline
\end{tabular}

the NN classifier performs marginally better. The highest attainable accuracy for the test set against the full training set is $99.7 \%$ for the bi-class problem of Devanagari vs. Kannada with a NN classifier while the lowest attainable, with the same classifier, is $97.3 \%$ for Odiya and Tamil combination. It could be noted that, the accuracies of the test set against the prototype set is also quite high, though trailing behind the full training set. This could be owing to the following reasons. Since we have tried to attain $100 \%$ accuracy for the prototype selection process, there is every likelihood that to attain that accuracy, the spurious patterns have also come into the prototype set. Thus, eliminating such out-lier patterns by the technique proposed by Susheela Devi et. al. could be of help. We could also terminate the process a little earlier than attaining $100 \%$ accuracy and see how such a prototype set behaves. The good bi-class accuracies obtained indicate that the five classes are well separated in the feature space.

Many official documents in India contain three scripts. They are Roman, Devanagari and the official script of the state, where the document is used. In the second series of experiments therefore, we have attempted discrimination between three scripts, out of which two are Roman and Devanagari. Based on the experiments on pairs of scripts, we expected good separation between scripts in a tri-script scenario using both of the LD and NN classifiers. The results of such experiments are presented in Tables 2 and 3 for LDC and NNC, respectively. The test sets have again been tested for their recognition accuracies against the train and the prototype sets.

The recognition accuracies are generally good. The discrimination between Tamil and combined Devanagari and Roman is relatively low, at $96.4 \%$ for the full train set and at $94.4 \%$ for prototype set. Here too, the recognition accuracies obtained with the prototype set closely follows the accuracies with the train set. When we compare the results presented in Table 3 (obtained from NNC) with those in Table 2 (obtained from LDC), NN classifier is observed to fare better than the LDC. This is in confirmation with the results for bi-class experiments. 
Table 2. Average recognition accuracies for script triplets comprising of Roman (R), Devanagari(D) and Indian local scripts(L). Here L/DR means the average recognition accuracy of the local script against the combined Devanagari and Roman scripts. Values shown are percentages for Gabor feature with LDC as the decision maker.

\begin{tabular}{|c|c|c|c|c|c|c|}
\hline \multirow{2}{*}{} & \multicolumn{3}{|c|}{ TRAIN } & \multicolumn{3}{|c|}{ PROTOTYPE } \\
\cline { 2 - 6 } & $\mathrm{K}$ & $\mathrm{O}$ & $\mathrm{T}$ & $\mathrm{K}$ & $\mathrm{O}$ & $\mathrm{T}$ \\
\hline $\mathrm{L} / \mathrm{DR}$ & 98.7 & 97.3 & 96.4 & 98.4 & 97.1 & 94.4 \\
\hline $\mathrm{D} / \mathrm{RL}$ & 98.7 & 99.0 & 98.6 & 97.6 & 98.7 & 98.3 \\
\hline $\mathrm{R} / \mathrm{DL}$ & 98.9 & 97.8 & 98.2 & 98.7 & 96.9 & 97.7 \\
\hline
\end{tabular}

Table 3. Tri-script recognition rate (in percentage) for the scripts of Kannada (K), Odiya (O) and Tamil (T) with Roman and Devanagari. The Gabor features have been used with NN classifier, against both the training and prototype sets.

\begin{tabular}{|r|c|c|c|c|c|c|}
\hline \multirow{2}{*}{} & \multicolumn{3}{|c|}{ TRAIN } & \multicolumn{3}{c|}{ PROTOTYPE } \\
\cline { 2 - 7 } & $\mathrm{K}$ & $\mathrm{O}$ & $\mathrm{T}$ & $\mathrm{K}$ & $\mathrm{O}$ & $\mathrm{T}$ \\
\hline Roman & 98.9 & 96.9 & 97.5 & 98.8 & 96.1 & 96.8 \\
\hline Devanagari & 98.7 & 98.9 & 98.3 & 98.1 & 97.4 & 97.5 \\
\hline Local & 99.3 & 98.9 & 97.3 & 99.3 & 97.6 & 96.1 \\
\hline Average & 99.0 & 98.2 & 97.7 & 98.7 & 97.0 & 96.8 \\
\hline
\end{tabular}

With the NN classifier too, Tamil script has the lowest average accuracy among the three local scripts, for both train and prototype sets.

We have compared our results with the earlier reported results of Pal \& Chaudhuri [10] and Padma \& Nagabhushan [11]3. Pal \& Chaudhuri have reported an accuracy of $97.2 \%$ for recognition of Devanagari words while Padma \& Nagabhushan have reported $97 \%$ for the same script. We achieve a minimum of $97.6 \%$ for the recognition of Devanagari words, against Kannada and Roman. This is the worst case we have when LDC is the decision maker and the test set is compared against the prototype set. The system performs better for combinations involving Kannada and Odiya as local scripts. The discrimination rate of Devanagari words against the other two is 98.7 and $98.3 \%$, for Odiya and Tamil as the local scripts, respectively, using the prototype set.

Thus a system, using LDC, employed for the discrimination between scripts in a tri-script scenario involving Kannada, Devanagari and Roman scripts would involve the separation of Roman at the first level with an accuracy of $98.9 \%$ and the separation between Kannada and Devanagari at the second level, with an accuracy of $99.5 \%$. This makes the overall accuracy of the system to be $98.4 \%$ with the train set. A similar arrangement with the prototype set gives $97.7 \%$. The average recognition accuracies for such a tri-script scenario, employing NNC as the decision maker, is $99.0 \%$ and $98.7 \%$ against the train and prototype sets, respectively. Thus our system, at all its configurations, outperforms the system

${ }^{3}$ Since we didn't have access to the databases used by Pal/Chaudhuri and Padma/Nagabhushan, the comparison is just numeric. 
Table 4. Recognition rate (in percentage) for a penta-class case involving all the five scripts, using the 36-dimensional Gabor feature with LD and NN classifiers

\begin{tabular}{|l|c|c|c|c|}
\hline \multirow{2}{*}{} & \multicolumn{3}{|c|}{ TRAIN } & PROTOTYPE \\
\cline { 2 - 5 } & LDC & NN & LDC & NN \\
\hline Roman & 95.8 & 96.1 & 94.4 & 94.9 \\
\hline Devanagari & 97.1 & 97.5 & 96.9 & 95.9 \\
\hline Kannada & 89.9 & 97.2 & 76.0 & 95.7 \\
\hline Odiya & 93.5 & 94.5 & 96.1 & 91.1 \\
\hline Tamil & 91.3 & 94.5 & 89.6 & 91.7 \\
\hline Average & 93.5 & 96.0 & 90.6 & 93.9 \\
\hline
\end{tabular}

reported by Padma \& Nagabhushan which has a maximum achievable accuracy of $96.7 \%$ for the same tri-script scenario.

Since our identification scheme depends on statistical rather than structural features, we have an advantage of taking any number of scripts and identifying them. On the observation that our feature classifier combinations are delivering us very good recognition accuracy, we tried to identify the scripts in a five-script scenario. In this case, any test sample is compared with the reference samples from all the classes by a NN classifier. The LDC classifier finds a discriminant function, which separates the script associated with the function, from rest four scripts. The test pattern is checked with all the discriminant functions for a score which measures its distance from the respective discriminant hyper-planes. The test pattern belongs to the script of the discriminant function yielding the maximum score. Table 4 presents the results of the script identification in this scenario. Consistent with our earlier recorded results, the combination of the NN classifier with the train set yields the best average result. Interestingly, while Kannada fares low with LDC, it is the Tamil and Odiya scripts that fare low with NN classifier.

\section{Conclusion and Discussion}

By testing with words of different font styles and sizes, we have shown that our identification scheme, utilizing the 36-dimensional feature, works consistently well for any combination of scripts. For example, the average recognition accuracy is $96 \%$ and the minimum accuracy for any script is $94.5 \%$, using nearest neighbour classifier employing the full training set (see Table 4). Similarly, the lowest mean identification rate is $97.7 \%$ (see Table 3) for the triscript scenario involving Tamil, Devanagari and Roman. The lowest recognition rate in bi-script documents is $97.3 \%$ for the case of Tamil against Odiya (see Table 1). However, a preliminary evaluation of the divergence of each of the features, shows that some features have better discriminating property than others. Thus a selected set of features would help in reducing the computation while enhancing the efficacy of the system. Our experiments indicate that such sets need to be uniquely selected for each of the cases separately, on a case by case basis. 
The prototype selection mechanism has been successfully able to reduce the train set by about $87 \%$. But the cardinality of the sets for different scripts shows the sets are highly skewed. Kannada gets represented by less than half the number of prototypes needed for the Odiya script. Similarly, Tamil script needs a little less than double the number of prototypes required for Kannada script. This gives us an impression that these two scripts, Odiya and Tamil, are relatively spread out in the feature space while the other three scripts form more compact clusters. When we look at the accuracy results recorded in Tables 1, 2, $3 \& 4$, we can observe that it is these two scripts which have been consistently faring slightly lower than others, in all cases. Despite all this, the results substantiate our assumption that the HVS inspired system is well suited for script identification in multi-script documents. However, this needs to be tested with other Indian and non-Indian scripts. Further, it will be interesting to compare the results against other features.

\section{Acknowledgment}

We would like to express our gratitude to Ms. G. Padmashree, Mr. Keshava and Mr. Ashwin for their help in collection and segmentation of the document images. We also thank the reviewers for improving the presentation of our results and conclusion. This work was supported by the fellowship grant of the Indian Institute of Science, Bangalore, India.

\section{References}

1. Spitz, A.L.: Determination of Script and Language Content of Document Images. IEEE transaction on Pattern Analysis and Machine Intelligence 19 (1997) 235-245

2. Hochberg, J., Kelly, P., Thomas, T., Kerns, L.: Automatic script identification from document images using cluster based templates. IEEE transaction on Pattern Analysis and Machine Intelligence 19 (1997) 176-181

3. Wood, S.L., Yao, X., Krishnamurthi, K., Dang, L.: Language identification for printed text independent of segmentation. In: Proc. of Intl. Conf. on Image Processing. (1995) 428-431

4. Tan, T.N.: Rotation invariant texture features and their use in automatic script identification. IEEE transaction on Pattern Analysis and Machine Intelligence 20 (1998) 751-756

5. Chaudhuri, A.R., Mandal, A.K., Chaudhuri, B.B.: Page layout analyser for multilingual indian documents. In: Proceedings of the Language Engineering Conference. (2002) 24-32

6. Pal, U., Chaudhuri, B.B.: Script line separation from Indian muli-script document. In: Proceedings of the International Conference on Document Analysis and Recognition. (1999) 406-409

7. Chaudhuri, S., Seth, R.: Trainable Script Identification Strategies for Indian languages. In: Proceedings of the International Conference on Document Analysis and Recognition. (1999) 657-660

8. Dhanya, D., Ramakrishnan, A.G., Pati, P.B.: Script identification in printed bilingual docuements. Sadhana 27 (2002) 73-82 
9. Pati, P.B., Raju, S.S., Pati, N.K., Ramakrishnan, A.G.: Gabor filters for document analysis in indian bilingual documents. In: Proc. of the Int. Conf. on Intelligent Sensing and Information Processing. (2004) 123-126

10. Pal, U., Sinha, S., Chaudhury, B.B.: Word-wise script identification from a document containing english, devanagari and telugu text. In: Proc. of National Conf. on Document Analysis and Recognition. (2003) 213-220

11. Padma, M.C., Nagabhushana, P.: Identification and separation of text words of kannada, hindi and english languages through discriminating features. In: Proc. of National Conf. on Document Analysis and Recognition. (2003) 252-260

12. Pati, P.B.: Indian Script Word Image Dataset, (www.ee.iisc.ernet.in/new/people/ students/phd/pati/)

13. Gabor, D.: Theory of communication. J. IEE (London) 93 (1946) 429-457

14. Daugman, J.: Uncertainty relation for resolution in space, spatial frequency and orientation optimized by two-dimensional visual cortical filters. J. Opt. Soc. Am. A 2 (1985) 1160-1169

15. Marcelja, S.: Mathematical description of the response of simple cortical cells. J. Opt. Soc. Am. 70 (1980) 1297-1300

16. Campbell, F.W., Robson, J.G.: Application of Fourier analysis to the visibility of gratings. J. Physiol. 197 (1968) 551-566

17. Morrone, M.C., Burr, D.C.: Feature detection in human vision: a phase dependent energy model. Proc. Roy. Soc. Lon.(B) 235 (1988) 221-245

18. Porat, M., Zeevi, Y.Y.: The generalized gabor scheme of image representation in biological and machine vision. IEEE transaction on Pattern Analysis and Machine Intelligence 10 (1988) 452-467

19. Jain, A.K., Farrokhnia, F.: Unsupervised texture segmentation using Gabor filters. Pattern Recognition 24 (1991) 1167-1186

20. Chan, W., Coghill, G.: Text analysis using local energy. Pattern Recognition 34 (2001) 2523-2532

21. Raju, S.S., Pati, P.B., Ramakrishnan, A.G.: Gabor filter based block energy analysis for text extraction from digital document images. In: Proc. of the First Int. Workshop on Document Image Analysis for Libraries (DIAL'04). (2004)

22. Devi, V.S., Murthy, M.N.: An incremental prototype set building technique. Pattern Recognition 35 (2002) 505-513 